\title{
Clinicopathological Significance of BMP7 Expression in Esophageal Squamous Cell Carcinoma
}

\author{
Koichi Megumi, MD, Sumiya Ishigami, MD, Yasuto Uchikado, MD, Yoshiaki Kita, MD, Hiroshi Okumura, MD, \\ Masataka Matsumoto, MD, Yoshikazu Uenosono, MD, Takaaki Arigami, MD, Yuko Kijima, MD, Masaki Kitazono, \\ MD, Hiroyuki Shinchi, MD, Shinichi Ueno, MD, and Shoji Natsugoe, MD
}

Department of Surgical Oncology, Digestive Surgery, Kagoshima University, Graduate School of Medicine, Kagoshima, Japan

\begin{abstract}
Background. Bone morphogenetic proteins (BMPs) are secreted signaling molecules belonging to the transforming growth factor- $\beta$ (TGF- $\beta$ ) superfamily of growth factors. Recent studies have shown that the influence of the expression of BMP7 was altered in several tumors. The purpose of the current study was to examine the expression of BMP7 in esophageal squamous cell carcinoma and to clarify the clinical impact of BMP7 expression in esophageal squamous cell carcinoma (ESCC).

Methods. A total of 180 patients with ESCC who underwent surgical resection from 1991 to 2004 were eligible for this study. The expression of BMP7 in esophageal tumor tissues was examined immunohistochemically.

Results. BMP7 expression was found in the cytoplasm of cancer cells. BMP7 positivity was observed in $61.7 \%$ of tumors. The BMP7-positive group had deeper progression, more advanced stages, and greater venous invasion than those without BMP7 expression ( $p<0.001, p<0.005$, and $p<0.0005$, respectively). In addition, expression of BMP7 correlated with poorer prognosis $(p<0.0005)$. Multivariate analysis showed that BMP7 expression status was an independent prognostic factor $(p<0.05)$.

Conclusions. Patients with expression of BMP7 in ESCC had high malignant potential. BMP7 could be a useful prognostic marker for patients with ESCC.
\end{abstract}

(C) The Author(s) 2011. This article is published with open access at Springerlink.com

First Received: 14 December 2010;

Published Online: 13 September 2011

K. Megumi, MD

e-mail: aircarter@goo.jp
Esophageal squamous cell carcinoma (ESCC) is aggressive in gastrointestinal tract carcinomas. Even when patients with ESCC undergo curative resection, postoperative relapse often occurs. Attempts to evaluate the group at high risk of recurrence have been made in respect to tumor aggressiveness. The tumor behavior of ESCC at the molecular level has been intensively analyzed.

Among the molecular factors available for possible evaluation of tumor aggressiveness, bone morphogenetic protein (BMP) is a member of the transforming growth factor (TGF)- $\beta$ superfamily known to regulate cell proliferation, apoptosis, and differentiation during human development. ${ }^{1,2}$ BMPs bind cell membrane type I and type II receptors of serine/threonine kinases, eliciting intracellular signaling via Smad1, 5, 8, and 9 proteins. ${ }^{3,4}$ Recent studies have shown that BMP7 maintains epithelial and endothelial phenotypes against epithelial-mesenchymal transition (EMT). ${ }^{5,6}$

BMP7 is present in cancers, including breast, prostate, and colon cancers, in which it is implicated in regulating cancer cell proliferation. ${ }^{7-9}$ Overexpression of BMP7 mRNA in colorectal cancer patients was significantly associated with poor prognosis and low overall survival. ${ }^{10}$ However, the expression of BMP7 in ESCC has not been evaluated.

In the present study, the BMP7 expression in surgical specimens of ESCC was examined to evaluate whether this molecule is useful to predict postoperative outcome.

\section{MATERIALS AND METHODS}

\section{Patients and Specimens}

There were 180 patients diagnosed with ESCC (163 males and 17 females) who underwent R0 
esophagectomy with lymph node dissection between 1991 and 2004 at Kagoshima University Hospital, Kagoshima, Japan. The median age of the patients was 65.2 (range, 39-84) years.

None of these patients underwent endoscopic mucosal resection, preoperative chemotherapy or radiotherapy, or had metachronous multiple cancer in other organs. Specimens of cancer tissue and noncancerous adjacent tissue were collected from patients after informed consent had been obtained in accordance with the institutional guidelines of our hospital. Using TNM classification of the International Union Against Cancer, ${ }^{11}$ all of the M1 tumors exhibited distant lymph node metastases. All patients were followed-up after discharge, with x-ray examination and tumor marker assays (squamous cell carcinoma antigen and carcinoembryonic antigen) performed every 1 to 3 months, computed tomography every 3 to 6 months, and ultrasonography every 6 months. Bronchoscopy and endoscopy were performed if necessary.

Postoperative follow-up data were obtained from all patients, with a median follow-up period of 39 (range, 1-137) months. According to TNM classification, 74 of the 180 patients had T1 tumors, 25 patients had T2 tumors, 78 patients had T3 tumors, and 3 patients had T4 tumors.

\section{Immunohistochemical Staining and Evaluation of BMP7 in ESCC}

Tumor samples were fixed with $10 \%$ formaldehyde in phosphate-buffered saline (PBS), embedded in paraffin, and sectioned into $4-\mu \mathrm{m}$-thick slices. They were deparaffinized in xylene and dehydrated with a series of graded ethanol. The endogenous peroxidase activity of specimens was blocked by immersing the slides in a $3 \%$ hydrogen peroxidase-methanol solution for $10 \mathrm{~min}$ at room temperature. After washing three times with PBS for 5 min each, the sections were treated with $1 \%$ bovine serum albumin for 30 min to block nonspecific reactions at room temperature. Then, the sections were incubated with primary antibody to BMP7 (1:200 R\&D System) at $4^{\circ} \mathrm{C}$ overnight and subsequently stained with secondary antibodies for $30 \mathrm{~min}$. The sections were incubated with avidin-biotin complex for $60 \mathrm{~min}$, and reactions were visualized using diaminobenzidine tetrahydrochloride for $2 \mathrm{~min}$. The sections were rinsed briefly in water and counterstained with hematoxylin for $30 \mathrm{~s}$. Normal human kidney was used as a positive (medullary rays) and negative (glomeruli) control to verify both BMP7 antibody specificity and staining quality (Fig. 1a). ${ }^{12}$ Immunohistochemistry was independently evaluated by two investigators (KM and SI) who
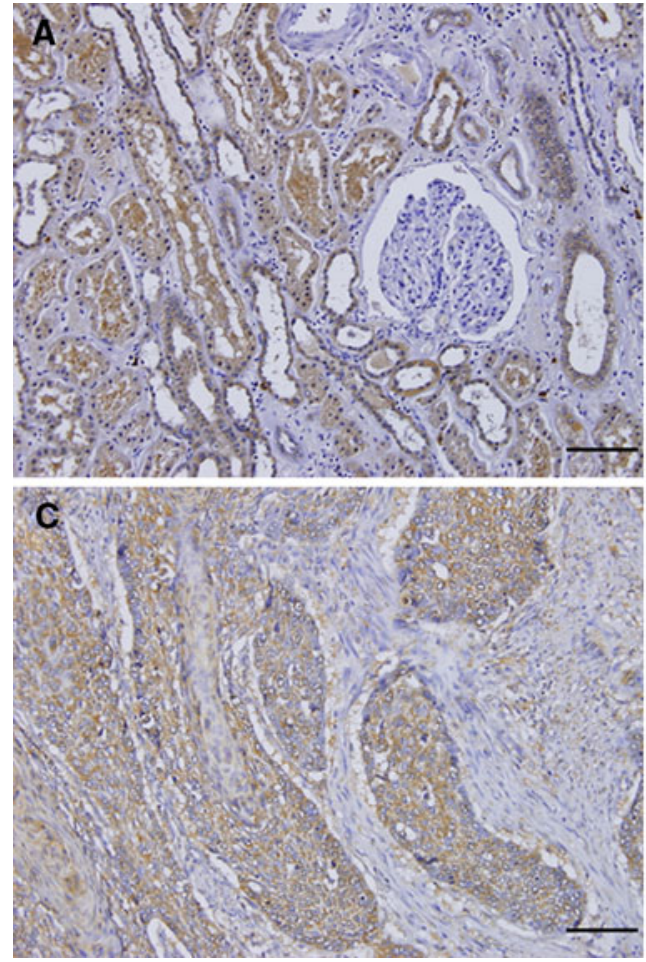

FIG. 1 Normal kidney section as a positive control for BMP7 staining. a BMP7 expression was seen in medullary rays and not seen in glomeruli $(\times 200)$. Expression of BMP7 in ESCCs. b Negative

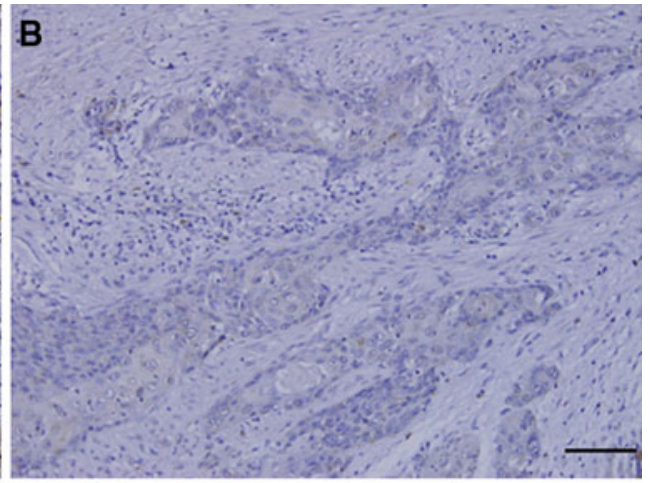

expression of BMP7 $(\times 200)$. c Positive expression of BMP7 was detectable in cytoplasm and membranes $(\times 200)$. Scale bar $100 \mu \mathrm{m}$ 
had no information about the patients' clinical data. In cases in which the immunohistochemical evaluation of the two observers differed, slides were evaluated by a third observer (SN).

To evaluate the expression of BMP7, ten fields (within the tumor and at the invasive front) were selected and expression in 1,000 tumor cells (100 cells/field) was evaluated using high-power $(\times 200)$ microscopy. Expression of BMP7 was assessed using the proportion of positive cells and using intensity. At first, we classified each sample into one of three categories in terms of the proportion of positive cells: $1+, 0-29 \% ; 2+, 30-59 \%$; and $3+, \geq 60 \%$ positive cells. In addition, we classified each sample into one of three categories according to the intensity: $1+$, weak; $2+$, moderate; and $3+$, strong. The tumors with strong staining at least $30 \%$ of the cells and moderate staining at least $60 \%$ of the cells were considered BMP7positive. We used this evaluation because of the heterogeneity of the ESCC samples and the fact that the deeper the tumors invaded, the stronger their intensity tended to become. We improved the evaluation method used by Liu et al. ${ }^{13}$

\section{Statistical Analysis}

Statistical analysis of group differences was performed using the $\chi^{2}$ test. The Kaplan-Meier method was used for survival analysis, and differences in survival were estimated by using the log-rank test. Prognostic factors were examined by univariate and multivariate analyses (Cox proportional hazards regression model). A $p$ value $<0.05$ was considered to indicate statistical significance. All statistical analyses were performed using the StatView for Windows software (Version 5.0, Abacus Concepts, Berkeley, CA).

\section{RESULTS}

\section{BMP7 Expression of ESCC}

BMP7 expression was identified in cellular membrane and cytoplasm of ESCC and was detected rarely in normal esophageal epithelium. Positivity of BMP7 expression was seen in $111(61.7 \%)$ of 180 patients (Fig. 1c).

\section{Relationship Between BMP7 Expression and Clinicopathological Features}

High-intensity BMP7 expression was detected more at deeper areas in ESCC. The correlations between BMP7 expression and clinicopathological characteristics are shown in Table 1. The BMP7-positive group had significantly
TABLE 1 Relationship between BMP7 expression and clinicopathologic findings

\begin{tabular}{|c|c|c|c|}
\hline & \multicolumn{2}{|c|}{ BMP7 expression } & \multirow[t]{2}{*}{$p$} \\
\hline & $\begin{array}{l}(-) \\
n=69\end{array}$ & $\begin{array}{l}(+) \\
n=111\end{array}$ & \\
\hline \multicolumn{4}{|l|}{ Total $(N=180)$} \\
\hline Age $($ mean $\pm \mathrm{SD})$ & $64.6 \pm 9$ & $65.6 \pm 9$ & 0.975 \\
\hline \multicolumn{4}{|l|}{ Gender } \\
\hline Male & 65 & 98 & 0.178 \\
\hline Female & 4 & 13 & \\
\hline \multicolumn{4}{|l|}{ Histology } \\
\hline Well & 19 & 36 & 0.588 \\
\hline Moderate & 34 & 53 & \\
\hline Poor & 9 & 22 & \\
\hline \multicolumn{4}{|l|}{$p^{T}$} \\
\hline $\mathrm{T} 1$ & 36 & 23 & $<0.0001$ \\
\hline $\mathrm{T} 2, \mathrm{~T} 3, \mathrm{~T} 4$ & 33 & 88 & \\
\hline \multicolumn{4}{|l|}{$\mathrm{p}^{\mathrm{N}}$} \\
\hline N0 & 34 & 43 & 0.171 \\
\hline N1 & 35 & 68 & \\
\hline \multicolumn{4}{|l|}{$\mathrm{p}^{\mathrm{M}}$} \\
\hline M0 & 55 & 78 & 0.06 \\
\hline M1 & 14 & 33 & \\
\hline \multicolumn{4}{|l|}{ Lymphatic invasion } \\
\hline Negative & 24 & 24 & 0.052 \\
\hline Positive & 45 & 87 & \\
\hline \multicolumn{4}{|l|}{ Venous invasion } \\
\hline Negative & 40 & 33 & 0.0002 \\
\hline Positive & 29 & 78 & \\
\hline \multicolumn{4}{|l|}{ Stage } \\
\hline I & 20 & 13 & 0.0012 \\
\hline IIA, IIB & 25 & 28 & \\
\hline III & 10 & 37 & \\
\hline IV & 14 & 33 & \\
\hline \multicolumn{4}{|c|}{ Locoregional recurrence } \\
\hline Negative & 65 & 4 & 0.027 \\
\hline Positive & 92 & 19 & \\
\hline
\end{tabular}

$S D$ standard deviation

deeper tumor invasion $(p<0.001)$, more advanced stages $(p=0.0012)$, more venous invasion $(p=0.0002)$, and more locoregional recurrence ( $p=0.027$ ) than the BMP7negative group. We had 23 locoregional recurrences during the period of this study: 3 were surgical anastomosis recurrences, and 20 were lymph node recurrences. Furthermore, we examined the relationship between BMP7 expression and locoregional recurrence; 19 of 23 patients with locoregional recurrence had BMP7-positive expression (Table 2). 
TABLE 2 Relationship between immunohistochemical staining for BMP7 expression and locoregional recurrence

\begin{tabular}{lccc}
\hline Recurrence site & Total & \multicolumn{2}{l}{ BMP7 expression } \\
\cline { 3 - 4 } & & $\begin{array}{l}\text { Positive } \\
(n=19)\end{array}$ & $\begin{array}{l}\text { Negative } \\
(n=4)\end{array}$ \\
\hline Lymph nodes & 20 & $17(85 \%)$ & $3(15 \%)$ \\
Surgical anastomosis & 3 & $2(66 \%)$ & $1(33 \%)$ \\
\hline
\end{tabular}

\section{BMP7 Expression and Patients' Survival}

The 5-year overall survival rate was significantly lower in patients with BMP7-positive expression than in those with negative expression ( $p=0.0004$; Fig. $2 \mathrm{a})$. In 77 patients who had no lymph node metastasis, 43 patients with BMP7-positive expression also had significantly lower 5-year overall survival rate than that of patients judged to have BMP7-negative expression $(p=0.0016$; Fig. 2b).

\section{Univariate and Multivariate Analysis of Survival}

Table 3 shows the results of univariate and multivariate analyses of the factors related to patient prognosis. Univariate regression analyses revealed that depth of tumor invasion, lymph node metastasis, pM status (distant lymph node metastasis), venous invasion, locoregional recurrence, and BMP7 expression significantly affected postoperative outcome. Multivariate analysis indicated that BMP7 expression was one of the independent prognostic factors ( $p=0.046)$, along with the depth of invasion $(p=0.019)$, and $\mathrm{pM}$ status (distant lymph node metastasis) $(p=0.009$; Table 3).

\section{DISCUSSION}

This is the first report to show an association between BMP7 expression and clinicopathological findings in ESCC. BMP7 positivity was observed in $61.7 \%$ of ESCC tumors, which was a higher incidence than in colorectal (41\%) and breast cancers $(46 \%) .{ }^{10,14}$

BMP7 expression have been reported to be involved in the growth of several cancer cells, such as osteosarcoma, malignant melanoma, prostate cancer, breast cancer, renal cell cancer, colorectal cancer, and gastric cancer, causing increased aggression or suppression. ${ }^{10,15-20} \mathrm{We}$ showed that BMP7 expression in ESCC was significantly associated with the tumor depth, stages, and venous invasion. Motoyama et al. investigated BMP7 mRNA in colorectal cancer and showed its significant association with tumor extension and liver metastasis. ${ }^{10}$ Aoki et al. reported immunohistochemical expression of BMP7 in gastric cancer was significantly related to the depth of tumors, nodal involvement, lymphatic invasion, and venous invasion. ${ }^{20}$ Those findings agree with our results. In this context, cancerous BMP7 positivity may reflect tumor aggressiveness.

In contrast, Kwak et al. reported that cancerous BMP7 expression correlated with better surgical outcome in renal cell carcinoma. ${ }^{15}$ Renal cells are used as a positive control of BMP7 expression; this contradictory finding supports organ-specificity. Aoki et al. also reported that BMP7 expression in gastric cancer was significantly higher in the well- and moderately differentiated histological group than in the poorly differentiated histological group. In contrast, in this study, we showed no relationship between BMP7 expression and tumor histological type; this contradictory findings supports tumor-specificity (histologic differences between squamous cell carcinoma and adenocarcinoma).
FIG. 2 a Overall 5-year survival curves of patients with esophageal cancer according to the expression of BMP7. Patients who were positive for BMP7 expression exhibited significantly poorer prognosis than those negative for BMP7 expression. b Overall 5-year survival curves of patients without lymph node metastasis in relation to BMP7 expression. Patients who were positive for BMP7 expression exhibited significantly poorer prognosis than those negative for BMP7 expression
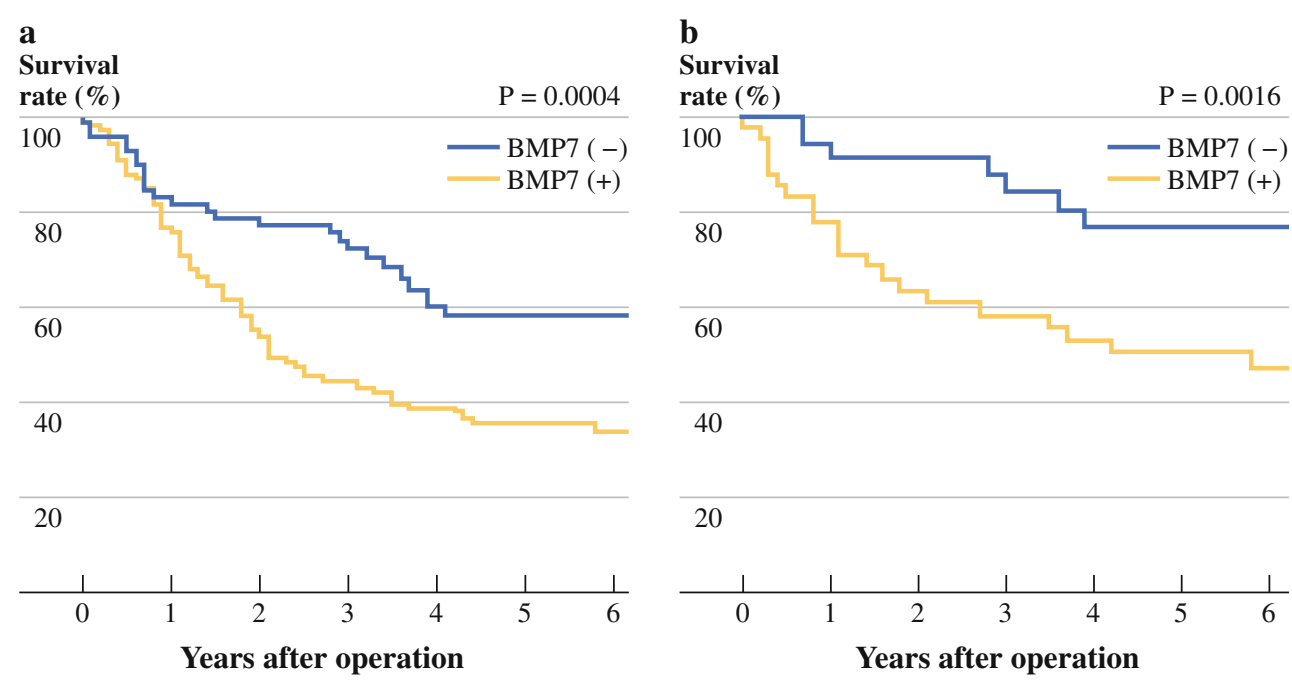
TABLE 3 Univariate and multivariate analysis of prognostic factors in ESCC

\begin{tabular}{lcccc}
\hline Factors & Univariate analysis & \multicolumn{2}{l}{ Multivariate analysis } \\
\cline { 3 - 5 } & $p$ & $p$ & \multicolumn{2}{c}{ Hazard ratio } \\
\hline pT $(1 / 2,3,4)$ (negative/positive) & $<0.0001^{*}$ & $0.019^{*}$ & 1.89 & $1.107-3.225$ \\
pN (negative/positive) & $0.0003^{*}$ & 0.377 & 1.269 & $0.748-2.15$ \\
Venous invasion (negative/positive) & $0.006^{*}$ & 0.622 & 1.121 & $0.71-1.769$ \\
pM (negative/positive) & $<0.0001^{*}$ & $0.009^{*}$ & 1.93 & $1.177-3.164$ \\
Locoregional recurrence (negative/positive) & $0.03^{*}$ & 0.587 & 1.162 & $0.673-2.008$ \\
BMP7 expression & $0.0004^{*}$ & $0.046^{*}$ & 1.61 & $1.008-2.576$ \\
\hline
\end{tabular}

$* p<0.05$

BMP7 expression by immunohistochemistry was significantly associated with lower recurrence-free survival in malignant melanoma and breast cancer. ${ }^{14,17}$ Our recent study showed BMP7 expression was significantly related to the locoregional recurrence. Those who had BMP7 expression tended to occur local lymph node relapse. Those findings might have important clinical significance. To determinate BMP7 expression status in primary ESCC could help to identify patients who are at high risk to develop early local lymph node recurrence.

In vitro, the BMP7 signaling pathway in malignant tumors has not been fully understood. From our results, in ESCC, these specific signals for BMP7 may correspond with tumor invasion in those signals that BMP7 stimulates.

In many cancers, TGF- $\beta$ is a protumorigenic factor that stimulates EMT. ${ }^{21-23}$ In contrast, BMP7 is a strong inducer of the reverse process (mesenchymal-epithelial transition; MET) during embryonic development. ${ }^{24-26}$ Moreover, BMP7 can inhibit TGF- $\beta$-induced fibrosis and counteracts TGF- $\beta$-induced EMT in normal renal epithelial cells. ${ }^{27-29}$ In contrast, in this study, overexpression of BMP7 in ESCC was significantly related to tumor aggressiveness. BMP7 might be related to tumor migration ability in ESCC. We need to find out more details about the cross-talk between BMP7 and TGF- $\beta$ signaling pathways in the context of EMT.

As a prognostic parameter, Aoki et al. reported that immunohistochemical BMP7 expression in gastric cancer patients was associated with poor overall survival. ${ }^{20}$ Moreover, overexpression of BMP7 mRNA was significantly associated with lower overall survival in colorectal cancer. $^{10}$

In this study, concerning the overall survival analysis, tumor depth, lymph node metastasis, venous invasion, distant lymph node metastasis, locoregional recurrence, and BMP7 expression were prognostic factors on univariate analysis. We showed that BMP7 expression was one of independent prognostic factors, along with tumor depth, and distant lymph node metastasis on multivariate analysis in accordance with results for other digestive tract cancers.
BMP7 expression could be used as a useful prognostic parameter predicting the survival of postoperative ESCC patients.

Various molecular markers have been shown to be associated with development and progression of ESCC, including cyclin D1, smad4, Fas, cyclin B1, CD151, Bmi1, and osteopontin. ${ }^{13,30-35}$ Naitoh et al. demonstrated that the survival rate for the patients with overexpression of cyclin D1 was very low even in the patients without lymph node metastasis. ${ }^{30}$ In this study, the 5-year overall survival rate for the patients with BMP7 positive expression was significantly worse than those with negative expression even in the patients without lymph node metastasis. BMP7 expression may be a useful marker for high-risk patients of ESCC without lymph node metastasis. This enables us to choose postoperative treatment, including chemotherapy.

Moreover, we showed that BMP7 expression tends to correlate with lymphatic invasion $(p=0.052)$. Thus, cancerous BMP7 expression in a biopsy may be informative to predict lymphatic invasion. When selecting whether to perform endoscopic resection for early ESCC, we can use cancerous BMP7 expression as a predictor of lymphatic invasion.

In conclusion, BMP7 expression in ESCC plays a critical role in tumor proliferation and invasion. Expression of BMP7 may be a predictor of poor overall survival. This independent molecular marker for high-risk patients could help us in our attempts to individualize patients' therapy.

OPEN ACCESS This article is distributed under the terms of the Creative Commons Attribution Noncommercial License which permits any noncommercial use, distribution, and reproduction in any medium, provided the original author(s) and source are credited.

\section{REFERENCES}

1. Patel SR, Dressler GR. BMP7 signaling in renal development and disease. Trends Mol Med. 2005;11:512-8.

2. Milan M. Sculpting a fly leg: BMP boundaries and cell death. Nat Cell Biol. 2007;9:17-8.

3. Attisano L, Wrana JL. Signal transduction by the TGF-beta superfamily. Science. 2002;296:1646-7. 
4. Massague J, Seoane J, Wotton D. Smad transcription factors. Genes Dev. 2005;19:2783-810.

5. Buijs JT, Rentsch CA, van der Horst G, et al. BMP7, a putative regulator of epithelial homeostasis in the human prostate, is a potent inhibitor of prostate cancer bone metastasis in vivo. Am J Pathol. 2007;171:1047-57.

6. Zeisberg EM, Tarnavski O, Zeisberg M, et al. Endothelial-tomesenchymal transition contributes to cardiac fibrosis. Nature. 2007;13:952-61.

7. Alamo EL, Rauta J, Kallioniemi A, et al. Bone morphogenetic protein 7 is widely overexpressed in primary breast cancer. Genes Chromosomes Cancer. 2006;45:411-9.

8. Miyazaki H, Watabe T, Kitamura T, Miyazono K. BMP signals inhibit proliferation and in vivo tumor growth of androgeninsensitive prostate carcinoma cells. Oncogene. 2004;23: 9326-35.

9. Beck SE, Jung BH, Del Rosario E, Gomez J, Carethers JM. BMPinduced growth suppression in colon cancer cells is mediated by p21(WAF1) stabilization and modulated by RAS/ERK. Cell Signal. 2007;19:1465-72.

10. Motoyama K, Tanaka F, Kosaka Y, et al. Clinical significance of BMP7 in human colorectal cancer. Ann Surg Oncol. 2008;15: 1530-36.

11. Sobin LH, Fleming ID. TNM classification of malignant tumors. 5th ed. Union Internationale Contre le Cancer and the American Joint Committee on Cancer. Cancer. 1997;80:1803-4.

12. Wang SN, Lapage J, Hirschberg R. Loss of tubular bone morphogenetic protein-7 in diabetic nephropathy. J Am Soc Nephrol. 2001;12:2392-9.

13. Liu WI, Guo XZ, Zeng MS, et al. Prognostic relevance of Bmi-1 expression and autoantibodies in esophageal squamous cell carcinoma. BMC Cancer. 2010;10:467.

14. Alarmo EL, Korhonen T, Huhtala H, et al. Bone morphogenetic protein 7 expression associates with bone metastasis in breast carcinomas. Ann Oncol. 2008;19:308-14.

15. Kwak C, Park YH, Kim IY, et al. Expression of bone morphogenetic proteins, the subfamily of the transforming growth factor$\beta$ superfamily, in renal cell carcinoma. J Urol. 2007;178:1062-7.

16. Sulzbacher I, Birner P, Lang S, et al. The expression of bone morphogenetic proteins in osteosarcoma and its relevance as a prognostic parameter. J Clin Pathol. 2002;55:381-5.

17. Rothhammer T, Wild PJ, Bosserhoff AK, et al. Bone morphogenetic protein 7 (BMP7) expression is a potential novel prognostic marker for recurrence in patients with primary melanoma. Cancer Biomark. 2007;3:111-7.

18. Masuda H, Hukabori Y, Yamanaka H, et al. Expression of bone morphogenetic protein-7 (BMp-7) in human prostate. Prostate. 2004;59:101-6.

19. Alamo EL, Rauta J, Kallioniemi A, et al. Bone morphogenetic protein 7 is widely overexpressed in primary breast cancer. Genes Chromosomes Cancer. 2006;45:411-9.
20. Aoki M, Ishigami S, Natugoe S, et al. Expression of BMP-7 in human gastric cancer and its clinical significance. $\mathrm{Br} J$ Cancer. 2011;104:714-8.

21. Thiery JP. Epithelial-mesenchymal transitions in tumour progression. Nat Rev Cancer. 2002;2:442-54.

22. Peinado H, Quintanilla M, Cano A. Transforming growth factor beta-1 induces snail transcription factor in epithelial cell lines: mechanisms for epithelial mesenchymal transitions. J Biol Chem. 2003;278:21113-23.

23. Piek E, Moustakas A, Kurisaki A, et al. TGF-(beta) type I receptor/ALK-5 and Smad proteins mediate epithelial to mesenchymal transdifferentiation in NMuMG breast epithelial cells. J Cell Sci. 1999;112:4557-68.

24. Dudley AT, Lyons KM, Robertson EJ. A requirement for bone morphogenetic protein-7 during development of the mammalian kidney and eye. Genes Dev. 1995;9:2795-807.

25. Luo G, Hofmann C, Bronckers AL, et al. BMP-7 is an inducer of nephrogenesis, and is also required for eye development and skeletal patterning. Genes Dev. 1995;9:2808-20.

26. Vukicevic S, Kopp JB, Luyten FP, et al. Induction of nephrogenic mesenchyme by osteogenic protein 1 (bone morphogenetic protein 7). Proc Natl Acad Sci U S A 1996;93:9021-6.

27. Simic P, Vukicevic S. Bone morphogenetic proteins in development and homeostasis of kidney. Cytokine Growth Factor Rev. 2005;16:299-308.

28. Wang S, Hirschberg R. BMP7 antagonizes TGF-beta-dependent fibrogenesis in mesangial cells. Am J Physiol Renal Physiol. 2003;284:F1006-13.

29. Zeisberg M, Hanai J, Sugimoto H, et al. BMP-7 counteracts TGFbeta1-induced epithelial-to-mesenchymal transition and reverses chronic renal injury. Nature. 2003;9:964-8.

30. Naitoh H, Shibata J, Hattori T, et al. Overexpression and localization of cyclin D1 mRNA and antigen in esophageal cancer. $A m$ J Pathol. 1995;5:1161-9.

31. Natsugoe S, Xiangming C, Aikou T, et al. Smad4 and transforming growth factor $\beta 1$ expression in patients with squamous cell carcinoma of the esophagus. Clin Cancer Res. 2002;8: 1838-42.

32. Chan KW, Lee PY, Srivastava G, et al. Clinical relevance of Fas expression in oesophageal squamous cell carcinoma. J Clin Pathol. 2006;59:101-4.

33. Takeno S, Noguchi T, Müller W, et al. Prognostic value of cyclin $\mathrm{B} 1$ in patients with esophageal squamous cell carcinoma. Cancer. 2002;94:2874-81.

34. Suzuki S, Miyazaki T, Kuwano H, et al. Prognostic significance of CD151 expression in esophageal squamous cell carcinoma with aggressive cell proliferation and invasiveness. Ann Surg Oncol. 2011;18:888-93.

35. Kita Y, Natsugoe S, Aikou T, et al. Expression of osteopontin in oesophageal squamous cell carcinoma. Br J Cancer. 2006;95: 634-8. 